Rev. Biol. Trop. 52(1): 187-199, 2004

www.ucr.ac.cr www.ots.ac.cr www.ots.duke.edu

\title{
Diversidad, abundancia y conjuntos ictiofaunísticos del sistema lagunar-estuarino Chantuto-Panzacola, Chiapas, México
}

Silvia Díaz-Ruiz ${ }^{1}$, Enrique Cano-Quiroga ${ }^{1}$, Arturo Aguirre-León ${ }^{2} \&$ Raúl Ortega-Bernal ${ }^{1}$

1 Lab. Ictiología y Ecología Costera, Depto. Hidrobiología, UAM-Iztapalapa, Apdo. Postal 55-535, México 09340, D.F. Fax: (5) 804 4738; sdr@xanum.uam.mx.

2 Lab. Ecología y Pesquerías, Depto. El Hombre y su Ambiente, UAM-Xochimilco, Apdo. Postal 23-181, México 04960, D.F. Fax: (5) 7235469; agla1269@cueyatl.uam.mx

Recibido 07-VIII-2001. Corregido 10-II-2003. Aceptado 11-III-2003.

\begin{abstract}
In the Chantuto-Panzacola system, Chiapas, México, a study of a fish community was carried out between March and November of 1997. From a total of 1456 fish collected, 31 species, 25 genera and 19 families had been identified. The diversity and abundance were high in March with $\mathrm{H}^{\prime}=2.67$ bits/ind, D = 3.89 sp/ind, biomass $1.88 \mathrm{~g} / \mathrm{m}^{2}$, density $0.25 \mathrm{ind} / \mathrm{m}^{2}$ and average weight $7.55 \mathrm{~g} /$ ind. Significant differences were found between the ecological parameters and the environmental variables of the ecosystem in the months of study (ANOVA and Tukey, $\mathrm{p}<0.05$ ). The multiple discriminant analysis showed that the temperature and the salinity was the most important factors in the variation of the community. The frequency analysis and spatial distribution of the fish allowed to define fish assemblages formed by several groups of species that inhabits the system. Nine dominant species were defined for the ecosystem, representing the $71 \%$ in number and the $76.4 \%$ in weight of the total catch, Achirus mazatlanus, Caranx hippos, Cathorops fuerthi, Centropomus robalito, Cichlasoma macracanthum, Cichlasoma trimaculatum, Gobiomorus maculatus, Gobionellus microdon and Lile gracilis.
\end{abstract}

Key words: Diversity, abundance, discriminant analysis, fish assemblages, Chantuto-Panzacola system, Chiapas.

Los ecosistemas lagunares-estuarinos que se encuentran en las costas tropicales se caracterizan por una compleja estructura ecológica, debido a su gran variedad de hábitats, su alta diversidad biológica e importante productividad primaria (Day et al. 1989). Esta productividad se debe a los subsidios que reciben las lagunas a través de las descargas fluviales y los movimientos mareales, así como también de las áreas de vegetación costera circundante (manglares, pastos de pantano) y sumergida (pastos marinos, macroalgas), que determinan la magnitud de la producción secundaria (Soberón-Chávez et al. 1986). La producción secundaria de la comunidad de peces es de gran importancia en ecosistemas costeros, ya que las especies funcionan como reguladores energéticos, debido a su capacidad de desplazamiento dentro del ecosistema así como entre ecosistemas, lo que determina complejas interacciones biológicas entre los peces y el entorno físico-ambiental. Dichas interacciones reflejan patrones de utilización del sistema a través de sus ciclos de vida, lo cual modifica la diversidad, distribución, abundancia y frecuencia de las poblaciones, de manera espacial y temporal (Yáñez-Arancibia et al. 1993, AyalaPérez et al. 2003, Díaz-Ruíz et al. 2003).

El sistema lagunar Chantuto-Panzacola en la costa de Chiapas, se considera de alto valor por su potencial biológico y pesquero. Es un área donde la actividad pesquera artesanal es 
intensa y dirigida principalmente al camarón (Anónimo 1994). La pesca de escama incluye róbalo (Centropomus nigresens, C. robalito), lisa (Mugil cephalus, M. curema) y mojarra (Cichlasoma macracanthum), las cuales son especies para consumo local. Por estas características, Chantuto-Panzacola representa un área prioritaria de evaluación ecológica de sus recursos bióticos, pues se desconocen aspectos básicos de su ecología, como la diversidad, los patrones de distribución y abundancia de la comunidad de peces, así como su comportamiento ambiental; factores que es necesario analizar para plantear alternativas de conservación y uso racional de las especies.

Diversos estudios han evaluado la fauna de peces de las costas norte y centro del Pacífico de México, entre los que se encuentran Ramírez-Hernández y Páez (1965), YáñezArancibia (1978), Amezcua Linares (1985), Pérez-Mellado y Findley (1985), Álvarez Rubio et al. (1986), Aceves-Medina et al. (2003), Gallardo-Cabello et al. (2003), y Ponce-Díaz et al. (2003). Para la región del Pacífico sur, los trabajos sobre estructura y función de las comunidades de peces son escasos. No obstante, existen algunos estudios que contienen información valiosa sobre ictiofauna de las costas del Golfo de Tehuantepec, como los de Ramírez-Hernández et al. (1964), Chávez (1979), Castro-Aguirre (1982), Acal y Arias (1990), Bianchi (1991) y Tapia-García (1998). Con base en estos estudios, es posible precisar que sólo algunos se refieren a esta región costera en particular, evidenciando la escasez de información sobre composición, diversidad, distribución y abundancia de la ictiofauna de los sistemas lagunares de Chiapas y la plataforma continental adyacente.

El presente estudio representa al parecer, la primera información sobre la comunidad de peces del sistema lagunar-estuarino ChantutoPanzacola en Chiapas. Por lo tanto, se plantea como primer objetivo, analizar la estructura de la ictiofauna a través de la diversidad, distribución y abundancia y su relación con algunas variables fisicoquímicas y en segundo lugar, definir especies dominantes y conjuntos ictiofaunísticos del sistema.

\section{MATERIALES Y MÉTODOS}

Área de estudio: El sistema lagunar-estuarino Chantuto-Panzacola se localiza en el Pacífico mexicano, en la costa sur de Chiapas, entre los $92^{\circ} 45^{\prime}$ y $92^{\circ} 55^{\prime} \mathrm{N}$ y los $15^{\circ} 09^{\prime}$ y $15^{\circ} 17^{\prime} \mathrm{W}$ (Fig. 1). El clima es tropical Am (f)w, cálido húmedo, lluvioso en verano y seco en invierno. Se reconocen dos épocas climáticas en la región: lluviosa de mayo a octubre y seca de noviembre a abril. El sistema tiene una extensión de $31.6 \mathrm{~km}^{2}$ y esta conformado por cinco lagunas principales: Chantuto, Campon, Teculapa, Cerritos y Panzacola. Se conecta con el mar a través de la Boca San Juan (BSJ) y recibe la descarga de

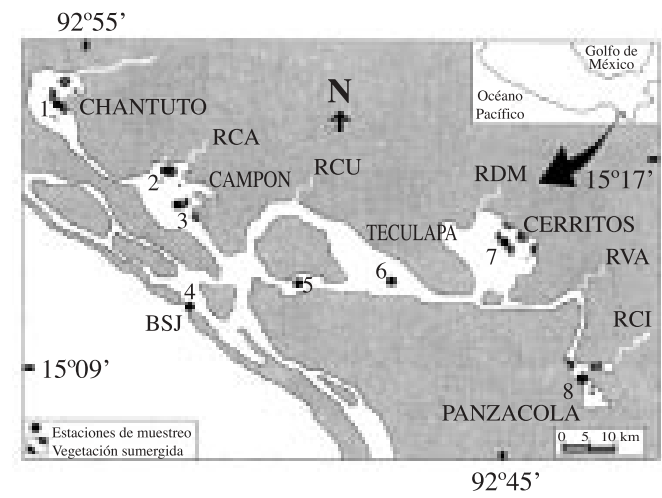

Fig. 1. Ubicación de Chantuto-Panzacola, Chiapas. La figura muestra la ubicación de las estaciones de muestreo, las características fisiográficas principales en el área y los ríos asociados con el sistema: Boca San Juan (BSJ), Río San Nicolás (RSN), Río Cuilapa (RCU), Río Sesecapa (RS), Río Ulapa (RU), Río Doña María (RDM) y Río Cintalapa (RCI).

Fig. 1. Location of Chantuto-Panzacola, Chiapas. The figure shows the sampling stations, the principal phisiographic characteristics and the associated rivers: San Juan Inlet (BSJ), San Nicolas River (RSN), Cuilapa River (RCU), Sesecapa River (RS), Ulapa River (RU), Doña María River (RDM) and Cintalapa River (RCI). 
varios ríos de pequeño caudal denominados: San Nicolás (RSN), Cacaluta (RCA), Cuilapa (RCU), Doña María (RDM) y Cintalapa (RCI). Existen diferentes ambientes en el ecosistema, como pantanos de manglar salobre y de baja salinidad, vegetación emergente y sumergida (Eichornea crassipes, Nymphaea ampla, Pistia stratiodes, Salvinia sp., Cyperus sp., Thypha dominguensis, Scirpus validus) (estaciones 1, 2, 3, 7, 8) y áreas de alta sedimentación. Amplios detalles de esta síntesis se encuentran en los trabajos de García (1988), Contreras-Espinosa e Ibarra-Obando (1997), Anónimo (1999).

Actividades de campo: La recolección de los peces se llevo a cabo en ocho estaciones de muestreo, durante los meses de marzo, mayo, julio y noviembre de 1997 (Fig. 1). Las capturas fueron diurnas en profundidades promedio de $1.80 \mathrm{~m}$, en cada estación se realizó un lance, utilizando un chinchorro playero (seine net) de $60 \mathrm{~m}$ de largo, caída de $2.0 \mathrm{~m}$, luz de malla de $1.9 \mathrm{~cm}$ en el copo y $1.5 \mathrm{~cm}$ en las alas. Las muestras fueron fijadas en formaldehído al $10 \%$, neutralizado con borato de sodio. En cada estación se registró la temperatura con un termómetro de cubeta (precisión $0.1^{\circ} \mathrm{C}$ ), la salinidad con un salinómetro YSI (precisión 0.01 $\%$, el oxígeno disuelto con un oxímetro YSI (precisión $0.01 \mathrm{mg} / \mathrm{l}$ ), la transparencia y profundidad de la columna de agua, con un disco de Sechii y una sondaleza, respectivamente. Los parámetros fisicoquímicos se compararon entre meses de estudio, utilizando análisis de varianza (ANOVA) con significancia $\mathrm{p}<0.05$ (Daniel 1997); de resultar diferencias significativas, se procedió a determinar entre que meses y parámetro ambiental utilizando la prueba de Tukey (Montgomery 1984).

Actividades de laboratorio: La determinación taxonómica se realizó empleando literatura específica del Pacífico y Golfo de Tehuantepec, principalmente los trabajos de Chirichigno et al. (1982), Fischer et al. (1995), Amezcua Linares (1996) y Castro-Aguirre et al. (1999). El orden sistemático se basó en el criterio de Nelson (1994). Se analizaron los siguientes parámetros ecológicos de la comuni- dad: diversidad (H') (Shannon y Wiener 1963), riqueza de especies (D) (Margalef 1969) y equitatividad (E) (Pielou 1966). Fue estimada la biomasa ( $\mathrm{g}$ peso húmedo/ $\mathrm{m}^{2}$ ), la densidad (ind $/ \mathrm{m}^{2}$ ) y el peso promedio (g/ind), éste último como una proporción del peso total y el total de individuos; además, se calculó la frecuencia de aparición y la dominancia de las especies (Yáñez-Arancibia et al. 1985). La diversidad, abundancia y peso promedio se compararon entre meses de estudio, utilizando ANOVA con significancia $\mathrm{p}<0.05$ (Daniel 1997); de resultar diferencias significativas, se determinó entre que meses y parámetros mediante la prueba de Tukey (Montgomery 1984). Se aplicó también, un análisis discriminante múltiple por pasos (forward), para identificar el subconjunto de variables que maximizaran las diferencias significativas ( $\mathrm{p}<0.05)$ entre los meses de estudio (Tatsuoka 1970, Ludwing y Reynolds 1988), con respecto a los factores ambientales y la variación de los parámetros ecológicos de la comunidad de peces. Con la información obtenida sobre la presencia/ausencia de las especies se efectuó un análisis de agrupamiento (cluster) para definir conjuntos ictiofaunísticos. Se utilizó el coeficiente de asociación de Jaccard, aplicando el paquete de computación ANACOM (De la Cruz-Agüero 1994).

\section{RESULTADOS}

Parámetros fisicoquímicos: Las variaciones espacio-temporales de los parámetros físicos y químicos se resumen en el Cuadro 1. La salinidad promedio más alta del sistema, se registró en las estaciones 4 y 5 con valores de $31.8 \%$ y $31.5 \%$ respectivamente, mientras que la más baja se presentó en la estación 8 con un promedio de $6.8 \%$. La salinidad promedio del agua presentó un intervalo de $26.5 \%$ en marzo, a $10.6 \%$ en noviembre. El ANOVA indicó diferencias entre los meses analizados, mientras que la prueba de Tukey señaló que las diferencias se presentaron entre marzo-noviembre y mayo-noviembre. Este sistema está 
CUADRO 1

Comportamiento espacial y temporal de parámetros ambientales del sistema lagunar-estuarino

Chantuto-Panzacola, Chiapas

TABLE 1

Spatial and temporal behavior of environmental parameters of the Chantuto-Panzacola system, Chiapas

\begin{tabular}{|c|c|c|c|c|c|c|c|c|c|}
\hline \multicolumn{10}{|c|}{ Estaciones } \\
\hline & 1 & 2 & 3 & 4 & 5 & 6 & 7 & 8 & Promedio \\
\hline \multicolumn{10}{|c|}{ Salinidad $\left(\%{ }_{00}\right)$} \\
\hline Marzo & 20 & 31 & 34 & 33 & 34 & 28 & 22 & 10 & 26.5 \\
\hline Mayo & 32 & 28 & 32 & 32 & 33 & 22 & 16 & 15 & 26.3 \\
\hline Julio & 4 & 20 & 26 & 32 & 32 & 11 & 2 & 2 & 16.1 \\
\hline Noviembre & 2 & 6 & 16 & 30 & 27 & 4 & 0 & 0 & 10.6 \\
\hline \multicolumn{10}{|c|}{ Temperatura $\left({ }^{\circ} \mathrm{C}\right)$} \\
\hline Marzo & 32.7 & 28.8 & 28.4 & 29.8 & 27.9 & 31.1 & 30.6 & 31.0 & 30.0 \\
\hline Mayo & 35.5 & 31.0 & 31.5 & 31.5 & 30.5 & 33.5 & 34.0 & 34.0 & 32.7 \\
\hline Julio & 30.5 & 31.0 & 31.0 & 31.5 & 31.0 & 29.0 & 29.0 & 30.0 & 30.4 \\
\hline Noviembre & 30.0 & 30.5 & 30.0 & 31.0 & 31.5 & 30.0 & 29.5 & 32.0 & 30.6 \\
\hline \multicolumn{10}{|c|}{$\mathrm{O}_{2}$ Disuelto (mg/l) } \\
\hline Marzo & 4.7 & 4.8 & 4.3 & 4.5 & 4.6 & 2.4 & 2.3 & 4.6 & 4.0 \\
\hline Mayo & 4.2 & 3.8 & 3.6 & 4.0 & 4.0 & 4.7 & 3.8 & 2.1 & 3.8 \\
\hline Julio & 4.1 & 3.7 & 1.6 & 3.2 & 3.3 & 0.8 & 1.4 & 1.3 & 2.4 \\
\hline Noviembre & 4.4 & 2.0 & 2.2 & 4.0 & 3.1 & 2.0 & 3.4 & 2.5 & 3.0 \\
\hline \multicolumn{10}{|c|}{ Transparencia (\%) } \\
\hline Marzo & 1.8 & 1.1 & 0.5 & 0.5 & 0.9 & 0.9 & 0.7 & 1.5 & 1.0 \\
\hline Mayo & 1.0 & 1.3 & 0.9 & 0.7 & 0.7 & 0.8 & 0.7 & 1.1 & 0.9 \\
\hline Julio & 0.9 & 0.8 & 0.6 & 0.6 & 0.4 & 0.4 & 0.6 & 1.8 & 0.8 \\
\hline Noviembre & 0.9 & 1.3 & 1.0 & 0.6 & 0.8 & 0.7 & 1.0 & 1.1 & 0.9 \\
\hline
\end{tabular}

influenciado principalmente por la entrada de agua salada del Golfo de Tehuantepec y por el agua dulce de los ríos locales. La temperatura del agua presentó los valores promedio más altos en las estaciones 1 y 6 con $32.2^{\circ} \mathrm{C}$ y $31.8^{\circ} \mathrm{C}$ respectivamente, mientras que los más bajos en las estaciones 3 y 5 con un promedio de $30.2^{\circ} \mathrm{C}$. En los meses de estudio la temperatura promedio fue mayor en mayo con $32.6^{\circ} \mathrm{C}$ y mínima en marzo con $29.9^{\circ} \mathrm{C}$. El ANOVA indicó diferencias entre los meses analizados, mientras que la prueba de Tukey señaló que se dieron entre marzo-mayo y mayo-julio.

El oxígeno disuelto tuvo su valor promedio más alto en la estación 4 con $4.4 \mathrm{mg} / \mathrm{l}$ y el más bajo en la estación 8 con $2.3 \mathrm{mg} / \mathrm{l}$. En el año, el valor promedio más alto fue registrado en marzo con $4.05 \mathrm{mg} / \mathrm{l}$ y él más bajo en julio con $2.32 \mathrm{mg} / \mathrm{l}$. El ANOVA indicó diferencias entre los meses analizados, mientras que la prueba de Tukey señalo que sólo fue entre marzo-julio. La transparencia de la columna de agua presentó valores altos en el sistema durante los meses de estudio, de 76 a $99 \%$. El ANOVA no indicó diferencias entre los meses analizados.

Estructura de la comunidad: En el sistema Chantuto-Panzacola, se obtuvo un total de 1456 peces, pertenecientes a 19 familias, 25 géneros y 31 especies. Las especies variaron de $26(81.3 \%)$ en el mes de marzo a 12 (37.5\%) en el mes de julio. Una lista sistemática de los peces se presenta en el Anexo 1.

Diversidad: Comparando la diversidad entre localidades de muestreo, los valores más altos de diversidad (H') y riqueza (D), se 
presentaron en la estación 8 con H' $=2.46$ bits/ind y $\mathrm{D}=2.86$ sp/ind. La equitatividad (E), tuvo el máximo valor en las estaciones 3 y 4 con $\mathrm{E}=1.0$ bits. En las estaciones 1, 3, 4, $6 \mathrm{y}$ 8 no se capturaron especies en algunos meses de muestreo, por lo que fueron registrados valores cero (Fig. 2). Comparando H' y D entre los meses de muestreo, se observó un comportamiento similar con los valores más altos en el mes de marzo, $\mathrm{H}^{\prime}=2.67$ bits/ind y $\mathrm{D}=3.89$ sp/ind y los mínimos en julio con $\mathrm{H}^{\prime}=2.09$ bits/ind y D = 2.24 sp/ind. El índice E, presentó un comportamiento inverso a los índices H'y $\mathrm{D}$, con el valor más alto $(\mathrm{E}=0.84$ bits $)$ en el mes de julio y él más bajo en mayo con $\mathrm{E}=$ 0.73 bits (Fig. 3). El ANOVA, no señaló diferencias entre los meses analizados para $\mathrm{H}^{\prime}$ y $\mathrm{E}$ ( $p>0.05$ ) mientras que si las hubo para D. La prueba de Tukey, mostró que se dieron entre marzo-julio y marzo-noviembre $(\mathrm{p}<0.05)$.

Abundancia: La figura 4 muestra el comportamiento de biomasa, densidad y peso promedio. Los valores más altos de biomasa y densidad se registraron en la estación 8 con $7.63 \mathrm{~g} / \mathrm{m}^{2}$ y $0.96 \mathrm{ind} / \mathrm{m}^{2}$. El peso promedio de los peces presentó su máximo valor en la estación 3 con $20.4 \mathrm{~g} /$ ind. En las estaciones 1, 3, 4 y 8 se registraron valores cero, debido a que no se capturaron individuos en algunos meses de muestreo. Comparando estos tres parámetros entre los meses de muestreo, se observa que los valores más altos se registraron en marzo con $1.88 \mathrm{~g} / \mathrm{m}^{2}, 0.25 \mathrm{ind} / \mathrm{m}^{2}$ y 7.55 $\mathrm{g} / \mathrm{ind}$, respectivamente; y los valores más bajos se presentaron en noviembre con 0.16 $\mathrm{g} / \mathrm{m}^{2}, 0.04 \mathrm{ind} / \mathrm{m}^{2}$ y $3.85 \mathrm{~g} /$ ind (Fig. 3). El ANOVA, no indicó diferencias entre los meses analizados para los parámetros antes descritos ( $\mathrm{p}>0.05)$.

Análisis discriminante: El análisis múltiple mostró que de cuatro variables significativas $(\mathrm{p}<0.05)$ incluidas en el modelo, dos fueron las más discrepantes como las funciones canónicas más importantes, la temperatura del agua con Lambda de Wilks de 0.177 y significancia $\mathrm{p}<0.0001$ y la salinidad con Lambda de 0.523 y $\mathrm{p}<0.007$. La correlación canónica del modelo para las variables incluidas fue de

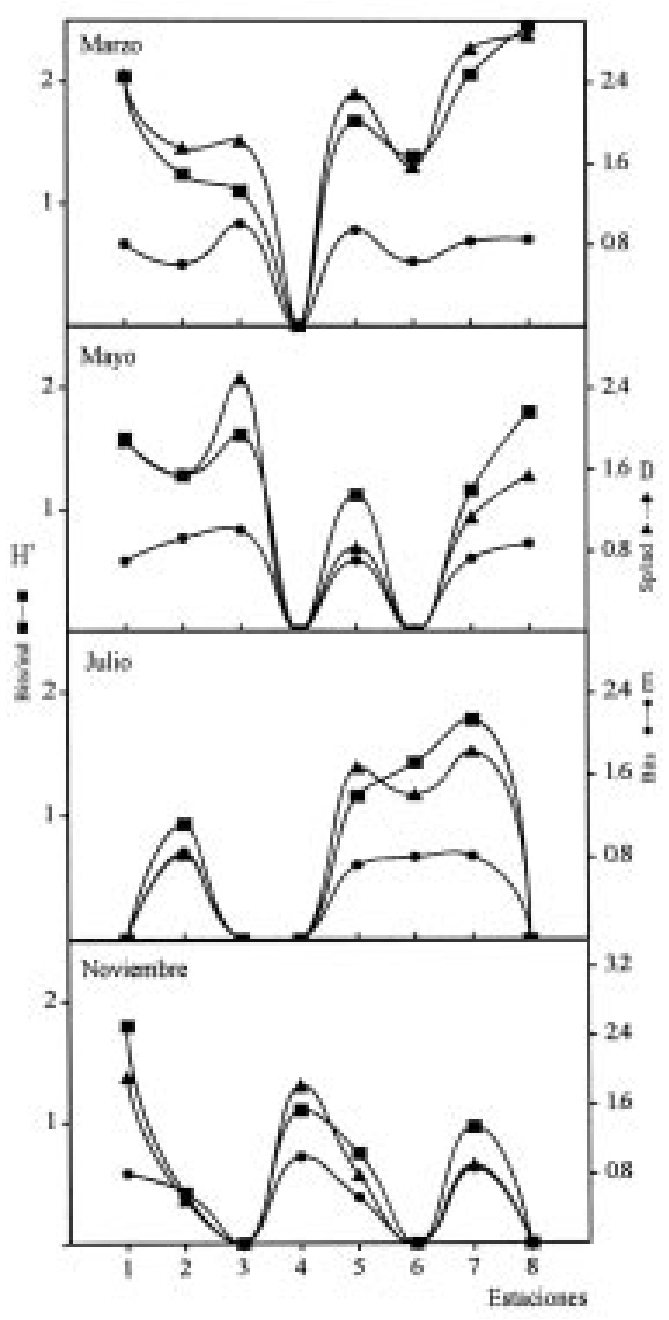

Fig. 2. Variación espacial de la diversidad en la comunidad de peces del sistema Chantuto-Panzacola. Diversidad numérica $\left(H^{\prime}=\right.$ bits/ind $)$, riqueza de especies $(\mathrm{D}=\mathrm{sp} / \mathrm{ind}) \mathrm{y}$ equitatividad $(\mathrm{E}=$ bits $)$.

Fig. 2. Spatial variation of diversity in the fish community of the Chantuto-Panzacola system. Diversity $\left(\mathrm{H}^{\prime}=\right.$ bits/ind), richness $(\mathrm{D}=\mathrm{sp} / \mathrm{ind})$ and evenness $(\mathrm{E}=$ bits$)$.

$r=0.813$. En el Cuadro 2, se muestra la matriz de clasificación para cada uno de los meses analizados de acuerdo con el análisis multivariado. La figura 5 muestra la distribución de observaciones correspondientes en función del espacio discriminante entre la primera y la segunda función. 

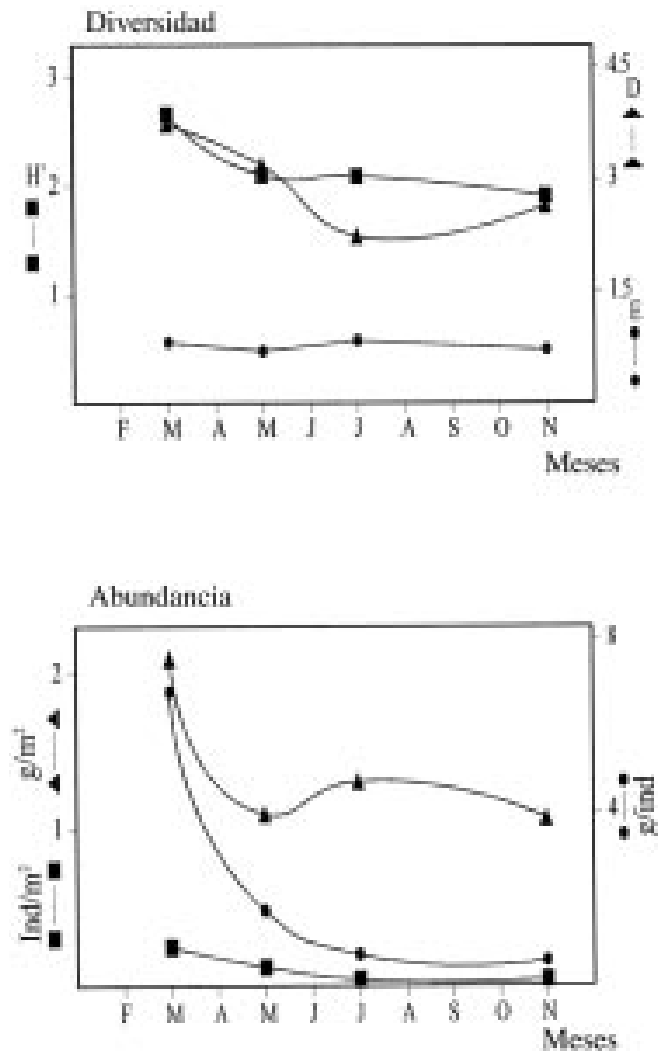

Fig. 3. Variación temporal de la diversidad y abundancia de la comunidad de peces en el sistema Chantuto-Panzacola. Diversidad numérica $\left(\mathrm{H}^{\prime}=\right.$ bits/ind), riqueza de especies $(\mathrm{D}=\mathrm{sp} / \mathrm{ind})$, equitatividad $(\mathrm{E}=$ bits $)$, biomasa $\left(\mathrm{g} / \mathrm{m}^{2}\right)$, densidad (ind $/ \mathrm{m}^{2}$ ) y peso promedio ( $\mathrm{g} / \mathrm{ind}$ ).

Fig. 3. Temporal variation of diversity and abundance in the fish community of the Chantuto-Panzacola system. Diversity $\left(H^{\prime}=\right.$ bits/ind $)$, richness $(\mathrm{D}=\mathrm{sp} / \mathrm{ind})$ and evenness $(\mathrm{E}=$ bits $)$, biomass $\left(\mathrm{g} / \mathrm{m}^{2}\right)$, density $\left(\mathrm{ind} / \mathrm{m}^{2}\right)$ and average weight $(\mathrm{g} / \mathrm{ind})$.

Especies dominantes: El análisis de abundancia numérica, peso y frecuencia, mostró que las especies dominantes de la comunidad son: Gobionellus microdon, Gobiomorus maculatus, Cichlasoma macracanthum, Lile gracilis, Cichlasoma trimaculatum, Centropomus robalito, Achirus mazatlanus, Cathorops fuerthi y Caranx hippos, las cuales representan el $71 \%$ de la abundancia numérica, el $76.4 \%$ de la captura total en peso y presentan las frecuencias más altas del sistema (Cuadro 3).

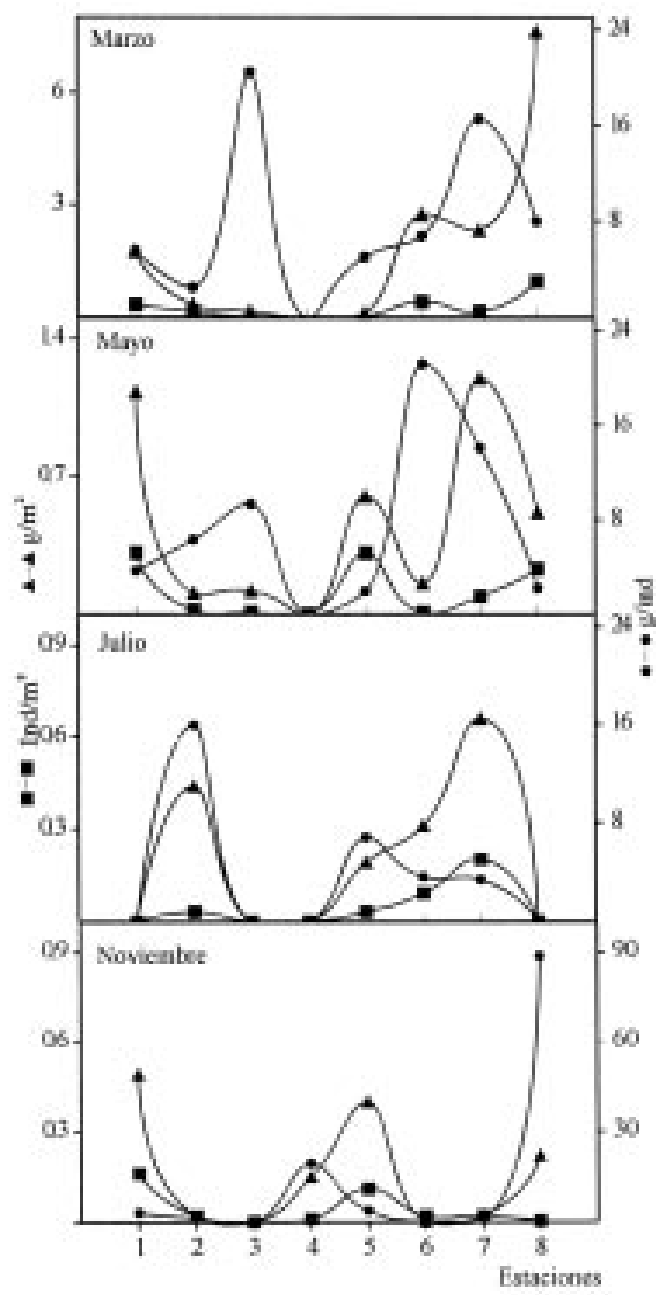

Fig. 4. Variación espacial de la abundancia en la comunidad de peces del sistema Chantuto-Panzacola. Biomasa $\left(\mathrm{g} / \mathrm{m}^{2}\right)$, densidad (ind $\left./ \mathrm{m}^{2}\right)$ y peso promedio ( $\left.\mathrm{g} / \mathrm{ind}\right)$.

Fig. 4. Spatial variation of abundance in the fish community of the Chantuto-Panzacola system. Biomass $\left(\mathrm{g} / \mathrm{m}^{2}\right)$, density (ind $/ \mathrm{m}^{2}$ ) and average weight ( $\mathrm{g} /$ ind).

Conjuntos ictiofaunísticos: Considerando la distribución espacial y temporal de los peces en Chantuto-Panzacola, se comparó la composición de la comunidad entre los meses estudiados para definir conjuntos de especies (Fig. 6). En esta figura se aprecia la formación de cuatro grupos de peces a niveles bajos de similitud. El conjunto I agrupa 14 especies, desde G. microdon hasta Gobiosoma etheostoma. 
CUADRO 2

Matriz de clasificación de las observaciones para las variables canónicas incluidas en el análisis discriminante multiple

TABLE 2

Classification matrix of the observations for the canonical variables included in the multiple discriminant analysis

\begin{tabular}{|c|c|c|c|c|c|}
\hline Meses & Porcentaje Correcto & Marzo & Mayo & Julio & Noviembre \\
\hline Marzo & 87.5 & 7 & 1 & 0 & 0 \\
\hline Mayo & 100.0 & 0 & 8 & 0 & 0 \\
\hline Julio & 75.0 & 0 & 1 & 6 & 1 \\
\hline Noviembre & 62.5 & 0 & 1 & 2 & 5 \\
\hline Totales & 81.3 & 7 & 11 & 8 & 6 \\
\hline
\end{tabular}

CUADRO 3

Distribución porcentual de la abundancia en número, peso y frecuencia de las especies dominantes del sistema lagunar-estuarino Chantuto-Panzacola, Chiapas

TABLE 3

Percentage distribution of the abundance in number, weight and frequency of the dominant species of the Chantuto-Panzacola system, Chiapas

\begin{tabular}{|c|c|c|c|c|c|}
\hline \multirow[t]{2}{*}{ Especies } & \multicolumn{2}{|c|}{ Individuos } & \multicolumn{2}{|c|}{ Peso (g) } & \multirow{2}{*}{$\begin{array}{c}\text { Frecuencia } \\
(\%)\end{array}$} \\
\hline & Total & $(\%)$ & Total & $(\%)$ & \\
\hline Gobionellus microdon & 210 & 14.4 & 762 & 8.8 & 43.8 \\
\hline Gobiomorus maculatus & 209 & 14.4 & 1133 & 13.0 & 28.1 \\
\hline Cichlasoma macracanthum & 122 & 8.4 & 1048 & 12.0 & 34.4 \\
\hline Lile gracilis & 105 & 7.2 & 429 & 4.9 & 18.8 \\
\hline Cichlasoma trimaculatum & 100 & 6.9 & 1119 & 12.9 & 31.3 \\
\hline Centropomus robalito & 80 & 5.5 & 594 & 6.8 & 28.1 \\
\hline Achirus mazatlanus & 76 & 5.2 & 53 & 0.6 & 28.1 \\
\hline Cathorops fuerthi & 72 & 5.0 & 878 & 10.1 & 21.9 \\
\hline Caranx hippos & 58 & 4.0 & 636 & 7.3 & 40.6 \\
\hline Totales & 1032 & 71.0 & 6653 & 76.4 & \\
\hline
\end{tabular}

Dentro de este grupo, se observa un subconjunto de 9 especies, donde quedan incluidas 7 de las especies dominantes definidas para el sistema (Cuadro 3), las cuales son componentes de origen marino y dulceacuícola que presentan amplia distribución y altas frecuencias en escala temporal. Un segundo subconjunto de 5 especies, agrupa igualmente componentes de ambos orígenes con frecuencias intermedias a bajas. Los conjuntos de especies II, III y IV y sus correspondientes subgrupos incluyen 18 especies, desde $C$. hippos hasta Oligoplites saurus, con frecuencias intermedias a muy bajas y en la mayoría de los casos, con distribución espacial restringida y presencia solo en alguno de los meses de estudio. El registro de especies en el año, mostró que en marzo y mayo se capturaron 26 y 20 especies respectivamente, mientras que en julio y noviembre fueron 12 y 14 especies, encontrándose variación en la abundancia de las especies dominantes en estos meses. 


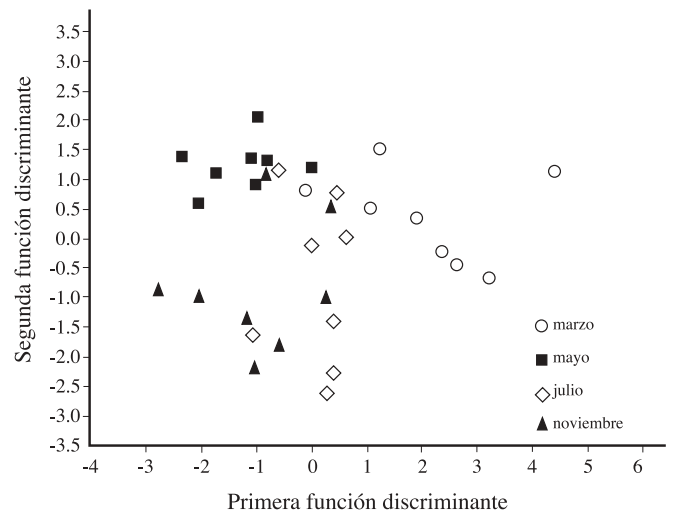

Fig. 5. Análisis discriminante múltiple de las variables ambientales y los parámetros ecológicos de la comunidad de peces en el sistema Chantuto-Panzacola.

Fig. 5. Multiple discriminant analysis of the environmental variables and ecological parameters of the fish community in the Chantuto-Panzacola system.

\section{DISCUSIÓN}

El análisis de la diversidad numérica $\left(\mathrm{H}^{\prime}\right)$ y la riqueza de especies (D) en el sistema, mostró que las variaciones espaciales y temporales son pequeñas, no obstante, están relacionadas con las condiciones hidrológicas y los diferentes ambientes presentes en el sistema. En Chantuto-Panzacola, se observó que los valores más altos de H' y D fueron registrados en las estaciones 3 y 8 , con presencia de vegetación sumergida. En los meses de estudio las variaciones de H'y D muestran relación con la temperatura y salinidad, registrándose la mayor diversidad en marzo, cuando la temperatura $\left(30.0^{\circ} \mathrm{C}\right)$ y salinidad $(26.5 \%$ \% son altas, disminuyendo hacia julio cuando los valores promedio de estos parámetros son de $30.4^{\circ} \mathrm{C}$ y $16.1 \%$. En marzo se capturó un mayor número de especies marinas como $\mathrm{A}$. mazatlanus, Anchoa lucida, C. hippos, $C$. robalito, Diapterus peruvianus, Himantura pacifica, Lutjanus argentiventris, O. saurus y Trinectes fonsecensis con mayor abundancia que en otros meses. Este comportamiento, sugiere que el ecosistema es utilizado por los peces optimizando las condiciones ambientales predominantes, como temperatura, disminu-

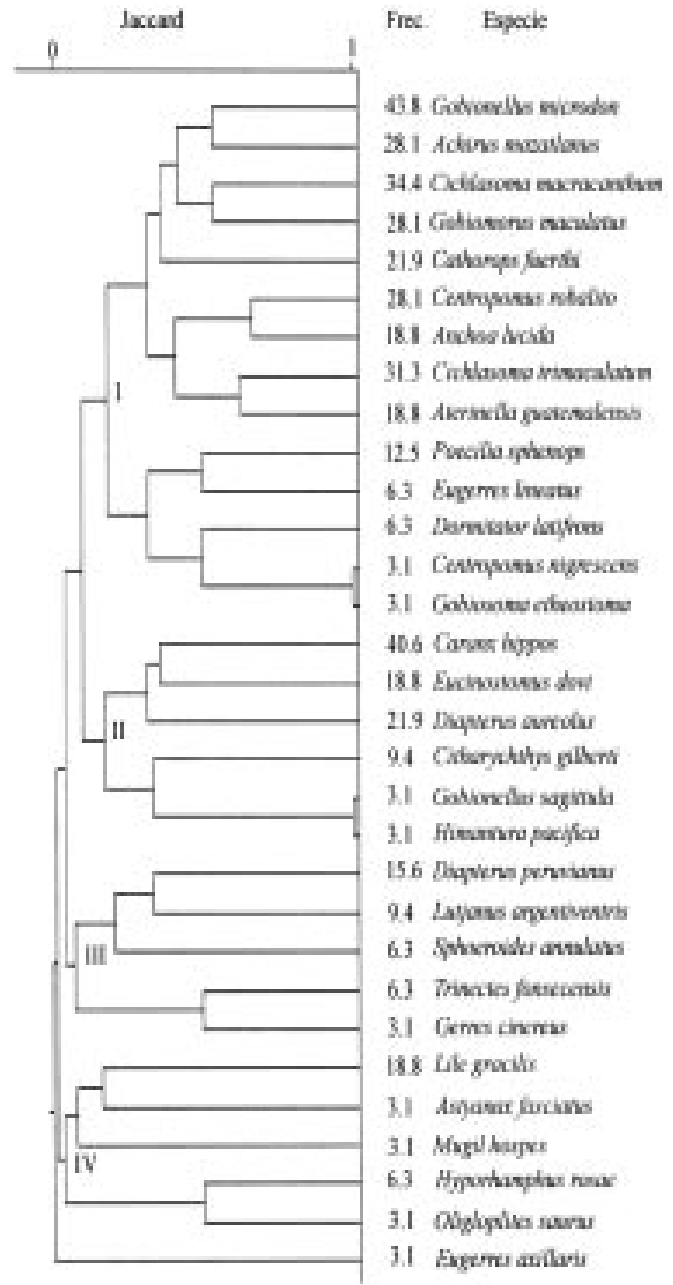

Fig. 6. Dendrograma de asociación de especies de peces para los meses de estudio en el sistema Chantuto-Panzacola.

Fig. 6. Cluster of association of fish species for the months of study in the Chantuto-Panzacola system.

ción de salinidad, descarga fluvial y presencia de vegetación sumergida, lo cual se refleja en los valores espacio-temporales de los parámetros comunitarios de las especies. De acuerdo con lo anterior, el análisis discriminante reforzó que las variables con mayor importancia en la variación de los parámetros ecológicos de la comunidad de peces, fueron la temperatura y la salinidad del sistema lagunar. En particular la variación de estos parámetros favorece la entrada de un mayor número de especies 
marinas como se observó en marzo y mayo. Un comportamiento similar ha sido observado por Soberón-Chávez et al. (1986), Yáñez-Arancibia et al. (1988), Aguirre-León et al. (1998), quienes encontraron que la diversidad numérica y riqueza de especies aumentan con la mayor variedad de hábitats en lagunas costeras tropicales. Lo mismo ocurre en sistemas costeros con gran heterogeneidad ambiental (Horn y Allen 1985, Hook 1991, Humphries et al. 1992 y Aguirre-León y Díaz-Ruiz 2000).

La variación de la abundancia mostró que los mayores valores se registraron en áreas caracterizadas por la presencia de vegetación sumergida (estaciones 1, 2, 7 y 8). La densidad y biomasa presentaron su valor más alto durante el mes de marzo y el menor en noviembre, lo cual esta relacionado con las variaciones de temperatura y salinidad, por la descarga de los ríos e intercambio de agua con el mar. El comportamiento de la biomasa y densidad en el sistema, se debe a la presencia de peces de tallas grandes observados durante marzo y mayo, sugiriendo que un mayor número de peces se presentan como preadultos y adultos utilizando las zonas de vegetación sumergida como áreas de maduración y alimentación en marzo y mayo, cuando los valores de temperatura y salinidad promedio son elevados. Por su parte, individuos juveniles utilizan la zona como área de crianza y protección durante noviembre, cuando la temperatura y salinidad promedio son los valores más bajos $\left(30.6^{\circ} \mathrm{C}\right.$ y $10.6 \%$ \% $)$. Numerosos estudios en otros ecosistemas lagunares costeros tropicales, han discutido la importancia de los hábitats con vegetación sumergida, su variación hidrológica y la relación con los cambios de biomasa de los peces costeros (Orth et al. 1984, Huh y Kitting 1985, Álvarez-Rubio et al. 1986, Rozas y Odum 1988, Lubbers et al. 1990, Sogard y Able 1991, Yáñez-Arancibia et al. 1993, Díaz-Ruíz et al. 2003).

Se considera que nueve especies son dominantes en el área de estudio, por su abundancia y distribución están relacionadas con las diferentes estrategias biológicas para utilizar los hábitats del sistema con fines alimenticios, re- productivos o de protección, como también con las variaciones ambientales (e.g. temperatura, salinidad, transparencia) del sistema a lo largo del tiempo (Horn y Allen 1985, Yáñez-Arancibia et al. 1988), las cuales son variables importantes en la distribución y abundancia de las especies dominantes en la comunidad de peces. Por lo anterior, la composición y dominancia de las especies en los conjuntos ictiofaunísticos varió en función de los meses y las características del ambiente. Durante el mes de marzo, seis especies presentaron la frecuencia más alta, destacando C. macracanthum, C. hippos, $G$. maculatus, A. mazatlanus, G. microdon y $C$. trimaculatum. Para el mes de mayo, C. hippos, G. microdon, C. macracanthum, G. maculatus y Eucinostomus dovi son las especies más frecuentes. Para estos dos meses la temperatura y salinidad del sistema son altas y es cuando se registra un incremento en la diversidad ( $\left.\mathrm{H}^{\prime}\right)$ por especies marinas. Para el mes de julio, tan sólo G. microdon, C. hippos y Diapterus aureolus, fueron las especies más frecuentes y en noviembre lo fueron A. mazatlanus, C. robalito, C. macracanthum y C. trimaculatum, las dos últimas de orígen dulceacuícola. Durante estos meses la salinidad y temperatura del sistema descienden, registrándose una disminución en la diversidad. La mayoría de las especies dominantes presentes en el sistema Chantuto-Panzacola, tienen amplia distribución geográfica en la costa del Pacífico mexicano y están bien adaptadas a las condiciones estuarinas de los sistemas costeros para realizar alguna parte de sus ciclos biológicos. Algunas de ellas como A. mazatlanus, C. robalito, C. fuerthi, L. gracilis y C. hippos, se han registrado en la plataforma continental adyacente de Oaxaca y Chiapas (Tapia-García 1998, Castro-Aguirre 1999).

El análisis de resultados muestra que debe continuarse el estudio del sistema lagunar Chantuto-Panzacola, ya que varias especies de peces son un recurso económico actual y otras representan un recurso potencial. Futuros estudios en este sentido junto con el conocimiento de los ciclos de vida de las especies de importancia ecológica y/o económica del sistema, 
permitirán comprender con mayor amplitud la estructura de las comunidades de peces en sistemas lagunares de Chiapas.

\section{AGRADECIMIENTOS}

Al Departamento de Hidrobiología de la Universidad Autónoma Metropolitana Iztapalapa y al Consejo Nacional de Ciencia y Tecnología (CONACYT, Convenio No. 400 200-5-4284 PT), por el apoyo institucional y económico para realizar este estudio. Los autores agradecen a Oswaldo Pérez-Solís y Edmundo Miranda-Blackmore su colaboración en las campañas de campo y laboratorio. A Samuel Marañón-Herrera por su asesoría en el análisis estadístico de datos.

\section{RESUMEN}

En el sistema lagunar-estuarino Chantuto-Panzacola, Chiapas, México, fue realizado un estudio ictiofaunístico entre marzo y noviembre de 1997. De un total de 1456 peces recolectados, se identificaron 31 especies, 25 géneros y 19 familias. La diversidad y abundancia presentaron sus valores más altos en el mes de marzo con $\mathrm{H}^{\prime}=2.67$ bits, $\mathrm{D}=$ $3.89 \mathrm{sp} / \mathrm{ind}$, biomasa $1.88 \mathrm{~g} / \mathrm{m}^{2}$, densidad $0.25 \mathrm{ind} / \mathrm{m}^{2}$ y peso promedio $7.55 \mathrm{~g} / \mathrm{ind}$. Se encontraron diferencias significativas (ANOVA y Tukey, $\mathrm{p}<0.05$ ) entre los parámetros ecológicos y las variables ambientales del ecosistema en los meses de estudio. El análisis discriminante múltiple, mostró que la temperatura y la salinidad son los factores más importantes en la variación de la comunidad. La frecuencia de aparición y distribución espacial de los peces, permitió definir conjuntos ictiofauníticos formados por diferentes grupos de peces que habitan el sistema. Se definieron nueve especies dominantes para el ecosistema, representando el $71 \%$ en número y el $76 \%$ en peso de la captura total. Estas especies son Achirus mazatlanus, Caranx hippos, Cathorops fuerthi, Centropomus robalito, Cichlasoma macracanthum, Cichlasoma trimaculatum, Gobiomorus maculatus, Gobionellus microdon y Lile gracilis.

\section{REFERENCIAS}

Aceves-Medina, G., R.J. Saldierna-Martínez \& E.A. González. 2003. Distribution and abundance of Syacium ovale larvae (Pleuronectiformes: Paralichthyidae) in the Gulf of California. Rev. Biol. Trop. 51: 561-570.
Aguirre-León, A., S. Díaz-Ruiz \& A. Bernal-Becerra. 1998. Ecología de Peces en Sistemas Fluvio-Deltaicos: Estudio para el Sistema Pom-Atasta en Campeche. Serie Académicos 28. Ciencias Biológicas y de la Salud. Universidad Autónoma Metropolitana-Xochimilco. México. 63 p.

Alvarez-Rubio, M., F. Amezcua-Linares \& A. YáñezArancibia. 1986. Ecología y estructura de las comunidades de peces en el sistema lagunar Teacapán-Agua Brava, Nayarit, México. An. Inst. Cien. Mar Limnol. 13(1): 185-242.

Amezcua-Linares, F. 1985. Recursos potenciales de peces capturados con redes camaroneras en la costa del Pacífico de México, pp. 39-94. In A. Yáñez-Arancibia (ed.). Recursos Pesqueros Potenciales de México: La Pesca Acompañante del Camarón. Universidad Nacional Autónoma de México-Instituto Nacional de la Pesca. México.

Amezcua-Linares, F. 1996. Peces demersales de la plataforma continental del Pacífico Central de México. Universidad Nacional Autónoma de México y Comisión Nacional para el Uso y Conservación de la Biodiversidad. México. 184 p.

Anónimo. 1994. Anuarios Estadísticos de Pesca 19781994. Secretaria de Pesca. México.

Anónimo. 1999. Programa de Manejo Reserva de la Biosfera La Encrucijada. Instituto Nacional de Ecología. México. 183 p.

Ayala-Pérez, L.A., J. Ramos Miranda \& D. Flores Hernández. 2003. La comunidad de peces de la Laguna de Términos: estructura actual comparada. Rev. Biol. Trop. 51: 783-794.

Bianchi, G. 1991. Demersal assemblages of the continental shelf and slope edge between the Gulf of Tehuantepec (México) and the Gulf of Papagayo (Costa Rica). Mar. Ecol. 73: 121-140.

Castro-Aguirre, J.L. 1982. Los peces de las Lagunas Oriental y Occidental, Oaxaca, México y sus relaciones con la temperatura y salinidad. II Análisis Multifactorial. An. Esc. Nal. Cien. Biol. 26: 85-100.

Castro-Aguirre, J.L., H. Espinosa Pérez \& J.J. SchmitterSoto. 1999. Ictiofauna Estuarino-Lagunar y Vicaria de México. Serie Biotecnológicas. Limusa. México. $705 \mathrm{p}$.

Chávez, E.A. 1979. Análisis de la comunidad de una laguna costera en la costa sur occidental de México. An. Inst. Cien. Mar Limnol. 6(2): 15-44.

Chirichigno, N., W. Fischer \& C.E. Naven. 1982. Catálogo de especies marinas de interés económico actual o potencial para América Latina. Parte 2. Pacífico 
Central y Suroriental. Roma, FAO/ PNUD, SIC/ 82/ 2: $1-588$.

Contreras-Espinosa, F. \& S. Ibarra-Obando 1997. Budgets for mexican coastal lagoons. Humid Pacific Coastal. Chantuto-Panzacola, Chiapas, pp. 47-50. In S.V. Smith, S. Ibarra-Obando, P.R. Boudreau \& V.F. Camacho-Ibar (eds.). Comparison of Carbon, Nitrogen and Phosphorus Fluxes in Mexican Coastal Lagoons. LOICZ Report Studies No. 10.

Daniel, W. 1997. Bioestadística: Base para el Análisis de las Ciencias de la Salud. Uthea. México. 878 p.

Day, J.W., C.A.S. Hall, W.M. Kemp \& A. Yáñez-Arancibia. 1989. Estuarine Ecology. Wiley. Nueva York. $576 \mathrm{p}$.

De la Cruz-Agüero, G. 1994. ANACOM: Sistema para el Análisis de Comunidades en computadoras personales. Versión 3.0. México. 99 p.

Díaz-Ruíz. S., M.A. Pérez Hernández \& A. Aguirre-León. 2003. Characterization of fish assemblages in a tropical coastal lagoon in the north west Gulf of Mexico. Ciencias Marinas 29(4B): 631-644.

Fischer, W., F. Krupp, W. Schneider, C. Sommer, K.E. Carpeter \& V.H. Niem. 1995. Guía FAO para la identificación de especies para los fines de la pesca. Pacífico Centro-Oriental. Volúmenes II y III. Vertebrados. Partes 1 y 2. FAO, Roma. pp. 647-1813.

García, E. 1988. Modificaciones al sistema de clasificación climática de Köeppen para adaptarlo a las condiciones de la República Mexicana. Instituto de Geografía. Universidad Nacional Autónoma de México. $346 \mathrm{p}$.

Gallardo-Cabello, M., E. Espino-Barr, F. GonzálezOrozco \& A. Garcia-Boa. 2003. Age determination of Anisotremus interruptus (Perciformes: Haemulidae) by scale reading, in the coast of Colima, Mexico. Rev. Biol. Trop. 51: 519-528.

Hook, J. 1991. Seasonal variation in relative abundance and species diversity of fishes in South Bay. Contrib. Mar. Sci. 32: 127-141.

Horn, M.H. \& L.G. Allen. 1985. Fish community ecology in southern California bays and estuaries. pp. 169190. In A. Yáñez-Arancibia (ed.). Fish Community Ecology in Estuaries and Coastal Lagoons: Towards an Ecosystem Integration. Universidad Nacional Autónoma de México. México.

Huh, S.H. \& C.L. Kitting. 1985. Trophic relationships among concentrated populations of small fishes in seagrass meadows. J. Exp. Mar. Biol. Ecol. 92: 29-43.
Humphries, P., I.C. Potter \& N.R. Loneragan. 1992. The fish community in the shallows of a temperate Australian estuary: relationships with the aquatic macrophyte Ruppia megacarpa and environmental variables. Estuar. Coast. Shelf Sci. 34: 325-346.

Lubbers, L., W.R. Boynton \& W.M. Kemp. 1990. Variations in structure of estuarine fish communities in relation to abundance of submerged vascular plants. Mar. Ecol. 65: 1-14.

Ludwing, J.A. \& J.F. Reynolds. 1988. Statistical Ecology. A Primer on Methods and Computing. Wiley. Nueva York. 337 p.

Margalef, R. 1969. Perspectives in Ecological Theory. University of Chicago, Chicago. $111 \mathrm{p}$.

Montgomery, D. 1984. Design and analysis of experiments. Williams. Nueva York. 538 p.

Nelson, I.S. 1994. Fishes of the World. Wiley. Nueva York. $600 \mathrm{p}$.

Orth, R.J., K.L. Heck Jr. \& J. van Montfrans. 1984. Faunal communities in seagrass bets: a review of the influence of plant structure and prey characteristics on predator-prey relationships. Estuaries 7 (4A): 339-350.

Pérez-Mellado, J. \& L.T. Findley, 1985. Evaluación de la ictiofauna acompañante del camarón comercial capturado en las costas de Sonora y norte de Sinaloa, México. pp. 201-253. In A. Yáñez-Arancibia (ed.). Recursos Pesqueros Potenciales de México: La Pesca Acompañante del Camarón. Universidad Nacional Autónoma de México-Instituto Nacional de la Pesca. México.

Pielou, E.C. 1966. The measurement of diversity in different types of biological collections. J. Theor. Biol. 13: $131-144$

Ponce-Díaz, G., S. Ortega-García \& S. HernándezVázquez. 2003. Lunar phase and catch success of the striped marlin (Tetrapturus audax) in sport fishing at Los Cabos, Baja California Sur, Mexico. Rev. Biol. Trop. 51: 555-560.

Ramírez Hernández, E. \& J. Páez. 1965. Investigaciones ictiológicas en las costas de Guerrero. I. An. Inst. Nal. de Invest. Biol. y Pesq. México. 1: 327-358.

Ramírez Hernández, E., G. Carrillo \& D. Lluch. 1964. Investigaciones ictiológicas de las costas de Chiapas. Lista de peces colectados en las capturas camaroneras (agosto-septiembre 1959, abril-mayo-junio 1960). Secretaría de Industria y Comercio, Dirección General de Pesca, México. Publicaciones del Instituto Nacional de Investigaciones Biológicas y Pesqueras. 5: 1-17. 
Rozas, L.P. \& W.E. Odum. 1988. Occupation of submerged aquatic vegetation by fishes: testing the roles of food and refuge. Oecology 77: 101-106.

Shannon, E.C. \& W. Weaver. 1963. The Mathematical Theory of Communication. University of Illinois. Urbana, $119 \mathrm{p}$.

Soberón-Chávez, G., A. Yáñez-Arancibia, P. Sánchez-Gil, J.W. Day \& L.A. Deegan. 1986. Relationships between physical/biological characteristics and fishery recruitment in tropical coastal ecosystems. pp. 5372. In A. Yáñez-Arancibia \& D. Pauly (eds.). Recruitment Processes in Tropical Coastal Demersal Communities. Ocean Science in Relation to living Resources, International Recruitment Project, IOCFAO-UNESCO Workshop OSLR/IREP Project. 44: $1-323$.

Sogard, S.M. \& K.W. Able. 1991. A comparison of eelgrass, sea lettuce macroalgae, and marsh creeks as habitats for epibenthic fishes and decapods. Estuar. Coast. Shelf Sci. 33: 501-519.

Tapia-García, M. 1998. El Golfo de Tehuantepec: El Ecosistema y sus Recursos. Universidad Autónoma Metropolitana-Iztapalapa. S \& G. México. 240 p.

Tatsouka, M.M. 1970. Discriminant Analysis. The study of group differences. IPAT, Champaign, III, $57 \mathrm{p}$.
Yáñez-Arancibia, A. 1978. Taxonomía, ecología y estructura de las comunidades de peces en lagunas costeras con bocas efímeras del Pacífico de México. Centro Ciencias del Mar y Limnología, Universidad Nacional Autónoma de México, Publicación Especial 2: 1306.

Yáñez-Arancibia, A., L. Lara-Domínguez, A. AguirreLeón, S. Díaz-Ruiz, F. Amezcua, D. Flores \& P. Chavance. 1985. Ecología de las poblaciones de peces dominantes en estuarios tropicales: Factores ambientales que regulan las estrategias biológicas y la producción. pp. 311-365. In A. Yáñez-Arancibia (ed.). Fish Community Ecology in Estuaries and Coastal Lagoons: Towards an Ecosystem Integration. Universidad Nacional Autónoma de México. México.

Yáñez-Arancibia, A., L. Lara-Domínguez, J.L. Rojas-Galaviz, P. Sánchez-Gil, J.W. Day Jr. \& C.J. Madden. 1988. Seasonal biomass and diversity of estuarine fishes coupled with tropical habitat heterogeneity (southern Gulf of México). J. Fish Biol. 33: 191-200.

Yáñez-Arancibia, A., A.L. Lara Domínguez \& J.W. Day Jr. 1993. Interactions between mangrove and seagrass habitats mediated by estuarine nekton assemblages: coupling of primary and secondary production. Hydrobiology 264: 1-12. 


\author{
Chantuto-Panzacola, Chiapas, México. \\ 1. Himantura pacífica (Beebe \& Tee-Van, 1941) \\ 2. Lile gracilis Castro Aguirre \& Vivero, 1990 \\ 3. Anchoa lucida (Jordan \& Gilbert, 1882) \\ 4. Astyanax fasciatus (Cuvier, 1819) \\ 5. Cathorops fuerthi (Steindachner, 1875) \\ 6. Hyporhamphus rosae (Jordan \& Gilbert, 1880) \\ 7. Poecilia sphenops (Valenciennes, 1846) \\ 8. Atherinella guatemalensis (Günther, 1864) \\ 9. Centropomus nigrescens Günther, 1868 \\ 10. Centropomus robalito Jordan \& Gilbert, 1882 \\ 11. Oligoplites saurus (Bloch \& Schneider, 1801) \\ 12. Caranx hippos (Linnaeus, 1766) \\ 13. Lutjanus argentiventris (Peters, 1869) \\ 14. Diapterus aureolus (Jordan \& Gilbert, 1882) \\ 15. Diapterus peruvianus (Cuvier \& Valenciennes, 1830) \\ 16. Eucinostomus dovi (Gill, 1863) \\ 17. Eugerres axillaris (Günther, 1864) \\ 18. Eugerres lineatus (Humboldt,1833) \\ 19. Gerres cinereus (Walbaum, 1792) \\ 20. Cichlasoma macracanthum (Günther, 1864) \\ 21. Cichlasoma trimaculatum (Günther, 1867) \\ 22. Mugil hospes Jordan \& Cuvier 1895 \\ 23. Dormitator latifrons (Richardson, 1844) \\ 24. Gobiomorus maculatus (Günther, 1859) \\ 25. Ctenogobius sagittula (Günther, 1861) \\ 26. Gobionellus microdon (Gilbert, 1891) \\ 27. Gobiosoma etheostoma (Jordan \& Starks, 1895) \\ 28. Citharichthys gilberti Jenkins \& Evermann, 1889 \\ 29. Achirus mazatlanus (Steindachner,1869) \\ 30. Trinectes fonsecensis (Günther, 1862) \\ 31. Sphoeroides annulatus (Jenyns, 1842)
}

ANEXO 1

Lista sistemática de las especies de peces capturadas en el sistema lagunar-estuarino 
